\title{
Disruptive Behaviors in Veterans Affairs Nursing Home Residents: How Different Are Residents with Serious Mental Illness?
}

\author{
John F. McCarthy, PhD,${ }^{* \dagger}$ Frederic C. Blow, PhD,${ }^{* \dagger}$ and Helen C. Kales, $M D^{*+\neq}$
}

OBJECTIVES: To determine the prevalence and correlates of behavior problems of residents of Veterans Affairs (VA) nursing homes and to compare residents with serious mental illness (SMI) with other resident groups.

DESIGN: This study combined cross-sectional resident assessments with administrative data for all residents in VA nursing homes. Multivariate ordinal logistic regression was used to evaluate associations between resident characteristics and problem behaviors.

SETTING: Nursing home care units in the VA healthcare system.

PARTICIPANTS: A total of 9,618 nursing home residents assessed as part of the VA's April 2001 national resident census.

MEASUREMENTS: The Patient Assessment Instrument assessed each resident's verbally disruptive, physically aggressive, and socially inappropriate behaviors in the prior 4 weeks. Functional limitations in eating, mobility, toileting, and transfer were assessed. Diagnoses were evaluated for the stay and up to 6 months before assessment.

RESULTS: Almost one-fifth (17.9\%) of residents received a diagnosis of SMI. Residents with SMI or dementia had greater behavior problems than residents with neither condition. Residents with SMI (and without dementia) exhibited more verbal disruption than residents with dementia (and without SMI), but the two subgroups did not differ in physically aggressive or socially inappropriate behavior.

CONCLUSION: Many VA nursing home residents have SMI. Their level of behavior problems is comparable with that of residents with dementia. Clinical practice and nursing home staff training must encompass geriatric mental

From the *Veterans Affairs (VA) National Serious Mental Illness Treatment Research and Evaluation Center, Health Services Research and Development, VA Ann Arbor Healthcare System, Ann Arbor, Michigan; ${ }^{\dagger}$ Division of Geriatric Psychiatry, University of Michigan, Ann Arbor, Michigan, and ${ }^{\ddagger}$ Geriatric Research Education and Clinical Center, VA Ann Arbor Healthcare System, Ann Arbor, Michigan.

Supported by VA Grant LIP 41-091 and VA HSR \& D Career Development Award RCD 00-037 (Dr. Kales). Presented at the annual meetings of the Gerontological Society of America (2003) and the American Association for Geriatric Psychiatry (2004).

Address correspondence to John F. McCarthy, PhD, HSR\&D Field Program, PO Box 130170, Ann Arbor, MI 48113.

E- mail: John.McCarthy2@med.va.gov health and behavior management to meet the needs of residents with SMI. J Am Geriatr Soc 52:2031-2038, 2004.

Key words: mentally ill persons; dementia; nursing homes; U.S. Department of Veterans Affairs

$\mathbf{F}^{\circ}$ or nursing home providers, resident disruptive behaviors pose challenges to quality of life, staff satisfaction and turnover, and quality of care. Staff may become distressed $^{1}$ and react negatively to aggressive patients, ${ }^{2}$ complicating treatment delivery. Disruptive behaviors require the focused attention of one or more staff members, reducing the number of staff available for other residents. Behavior problems also occur most frequently at busy times for staff, such as when assisting residents with activities of daily living (ADLs). ${ }^{3}$ Provider responses to problem behaviors include sedation, ${ }^{4}$ antipsychotic medications, physical restraints, ${ }^{5}$ and an unwillingness to admit residents who are expected to have significant behavior problems.

Nursing home administrators have cited concerns about potential behavior problems as a major factor affecting decisions about whether to admit individuals with serious mental illness (SMI), ${ }^{6}$ but these administrators have also reported that residents with SMI are basically similar in general behavior to the general residential population. Facility administrators' inconsistent reports regarding the behaviors of residents with SMI raise the possibility that stigma and anxieties associated with SMI may affect admission and care decisions.

Administrators may also be unwilling to admit individuals with SMI because nursing home staff receive little training in mental health or behavioral management. ${ }^{7,8}$ Lacking training, staff may rely on folk conceptions of mental illness, many of which are stigmatizing and inaccurate. ${ }^{7}$ Nursing directors perceive only limited competence on the part of staff at all levels in managing patients with behavioral disturbances, ${ }^{9}$ and this may heighten concerns surrounding the admission of patients with SMI.

The recognition of the substantial prevalence of mental illness in nursing homes and concerns regarding the "dumping" of individuals with SMI into nursing homes motivated the Omnibus Budget Reconciliation Act of 1987's 
Pre-Admission Screening and Annual Resident Reviews (PASARR). ${ }^{10}$ These regulations were intended to avoid inappropriate admissions to nursing homes of mentally ill patients who did not require nursing home level of services and to refer these individuals to psychiatric care centers. In 1996, Congress repealed the required annual resident reviews. ${ }^{11}$ Although the PASARR requirements have been credited with reducing the prevalence of residents with SMI and no medical comorbidities, ${ }^{12}$ they have also been widely criticized as ineffective. ${ }^{13,14}$ For example, half of surveyed nursing home administrators reported that they have had no effect on nursing home practices. ${ }^{15}$ Moreover, a regulatory emphasis on restricting admissions may obscure the fact that many individuals with SMI require the level of services that nursing homes provide.

Mental health policy makers are concerned that individuals with SMI may lack access to needed nursing home services. However, it is difficult to evaluate the assumption that individuals with SMI are more likely to be disruptive in nursing home settings. First, currently, there is only limited evidence regarding the prevalence and correlates of disruptive behaviors of nursing home residents with SMI. Second, few studies have examined how these residents compare with other subpopulations of nursing home residents.

Estimates of the prevalence of behavioral problems in nursing home residents vary considerably, in part because of differences in definition and measurement. Of nursing home residents in the Nursing Home Component of the 1996 Medical Expenditure Panel Study, 30.2\% exhibited at least one form of inappropriate or dangerous behavior. ${ }^{16}$ One study ${ }^{3}$ reported that $43 \%$ of cognitively impaired elderly nursing home residents had some reported disruptive behavior. Another study ${ }^{17}$ summarized survey data documenting some behavioral disturbance in $90 \%$ of residents. In some cases, the severity of behavioral syndromes in nursing homes has been reported to be equivalent to that encountered in psychiatric inpatients. ${ }^{17}$

Nursing home residents with mental illness may be more likely to have behavior problems, ${ }^{18,19}$ and mental illness is ubiquitous in nursing homes; $80 \%$ of residents have some diagnosable psychiatric disorders. ${ }^{20,21}$

In nursing homes, the most common mental illness is dementia. About $48 \%$ of nursing home residents have dementia. ${ }^{16,20}$ Problem behaviors may occur in $50 \%$ to $90 \%$ of individuals with dementia. ${ }^{22,23}$ Dementia is more familiar to nursing home staff than other mental illnesses, and staff may be more comfortable with residents with dementia than those with other forms of mental illness such as chronic schizophrenia. ${ }^{8}$

Behavior problems in residents with dementia have received substantial research and clinical attention. ${ }^{22}$ Indeed, for practical reasons, dementia is excluded from the set of mental illnesses that are evaluated in the PASARR requirements of the Omnibus Budget Reconciliation Act of $1987^{10}$ and that define Institutions for Mental Diseases. ${ }^{24}$ However, few studies have examined behavioral disturbances among residents with SMI including schizophrenia, bipolar disorder, and other psychoses.

Individuals with SMI have high needs for nursing home services, and despite potential access barriers, they are overrepresented in the long-term care population. ${ }^{25}$ Schizophrenia and other psychoses may be present in as many as
$13 \%$ of all U.S. nursing home residents, ${ }^{26}$ with wide variation in concentration across facilities. ${ }^{7,27}$

Research suggests high levels of impairment and behavior problems in nursing home residents with SMI. They have worse cognitive and functional impairment and more aggressive behaviors than do similarly diagnosed individuals in community settings. ${ }^{28}$ Indeed, in the frequency and severity of their verbally and physically aggressive behavior, elderly patients with schizophrenia in Veterans Affairs (VA) nursing homes may not differ from state psychiatric hospital inpatients. ${ }^{29}$

Few large studies have evaluated whether SMI is related to the likelihood of behavioral problems in nursing home residents when controlling for other patient characteristics. The two prior studies examining this issue yielded conflicting results. In nursing home residents included in the $\mathrm{Na}-$ tional Medical Expenditure Survey, a group of researchers ${ }^{30}$ found that having schizophrenia and other psychoses was associated with increased risk of behavior problems, controlling for age, sex, ADL dependency, and cognitive status, but with similar covariates, other researchers ${ }^{31}$ found no such association in a large sample of nursing home residents in Rhode Island.

Given the limited information available on the relative prevalence and severity of behavioral disturbances of nursing home residents with SMI compared with other resident subpopulations, it is difficult to evaluate the potential access barriers to nursing home care.

The purpose of this study was to examine diagnostic correlates of disruptive behaviors in nursing home residents in the VA national healthcare system. Three questions were evaluated.

1. What is the prevalence of SMI (defined as schizophrenia, bipolar disorder, or other psychosis) in nursing home residents in the VA national healthcare system?

2. What is the prevalence and severity of disruptive behaviors among these individuals?

3. With regard to disruptive behaviors, how do nursing home residents with SMI compare with residents with dementia, those with dementia and SMI, and those with neither condition?

\section{METHODS}

On April 1, 2001, 9,707 residents of VA nursing home care units were assessed using the semiannual long-term care Patient Assessment Instrument (PAI). The PAI was developed for long-term-care case-mix reimbursements based on Resource Utilization Groups. ${ }^{32}$ Data were collected at each facility by lead staff. Data were analyzed for 9,618 (99.1\%) of these individuals after excluding 44 residents who were comatose and 45 residents for whom there was no corresponding VA utilization and diagnosis data.

The PAI includes evaluations of dependency in four ADLs (eating, mobility, transfer, and toileting) and three behavioral problems (verbal disruption (e.g., yelling, baiting, threatening), physical aggression (assertive or combative to self or others with possibility of injury), and inappropriate behavior (disruptive, infantile, or socially inappropriate behavior (childish, repetitive, or antisocial 
physical behavior that creates disruptions with others)) during the 4 weeks before assessment. For each ADL, residents were rated on a 5-point scale, with higher values indicating greater frequency and dependence. Behaviors were rated, with higher values indicating greater frequency and unpredictability. Ratings were recoded so that values ranged from 0 (no such behavior in the prior 4 weeks) to 3 (unpredictable, recurring behavior at least once per week).

Diagnosis data in the VA's Patient Treatment File (PTF) for all VA health services utilization in the prior 6 months, including nursing home activity on April 1, 2001, were examined. The PTF is a national computerized administrative database containing information on patient characteristics (age, race, sex, and marital status) and including primary and secondary diagnoses associated with admissions. We assessed whether subjects received International Classification of Diseases, Ninth Revision, diagnosis codes for dementia (046.1, 290.0-290.4, 291.2, 292.82, 294.1, 331.0, 331.1, 331.7, 331.82, 331.9); schizophrenia, bipolar disorder or other psychoses/SMI (295.x, 296.0, 296.1, 296.4296.9, 297.x, 298.x); depression (296.2, 296.3, 296.5, 298.0, 311.0-311.9); and substance abuse/dependence (291.x, 292.x, 303.x, 304.x, 305.x). Dichotomous variables indicated the presence (coded as 1) or absence (0) of diagnoses for each condition.

From the PAI, nursing home resident age in years, sex ( male $=1$; female $=0)$, and number of days of nursing home stay at assessment (assessment date-admission date) were assessed.

An indicator of military service-connected disability, derived from VA administrative data, was also included. The Veterans Millennium Health Care and Benefits Act, enacted in 1999, included a mandate that the VA healthcare system provide nursing home and noninstitutional longterm care to veterans with at least a $70 \%$ service-connected disability status. Service connection at $70 \%$ or greater was coded via a dichotomous indicator $(1$ if $\geq 70 \%$ service connected, otherwise 0 ). This measure may be understood to encompass dimensions of access to care and illness severity and affiliation with the VA healthcare system.

For descriptive analyses, each resident was categorized as having SMI without comorbid dementia, having dementia without comorbid SMI, having SMI and dementia, and having neither condition. Resident and stay characteristics (age, sex, service connection level, functional status limitations, depression, substance abuse, and days of nursing home stay) were compared across diagnosis groupings.

The associations between patient psychiatric morbidities and three dimensions of behavior problems (verbal disruptions, physical aggression, and inappropriate behavior) were examined. These were each measured as ordinal variables. In addition to assessing the relative prevalence of these behaviors via descriptive analyses, multivariate modeling techniques were applied to assess the relative effects of the presence of SMI and of dementia, while controlling for relevant covariates. Given that the three dependent measures are evaluated on ordinal scales, ordinal logistic regressions, which is a proportional odds model using maximum likelihood estimation, were performed. ${ }^{33}$ SAS software (SAS Institute, Inc., Cary, NC) was used for all statistical analyses.

\section{RESULTS}

The VA nursing home resident population is predominantly $(96.3 \%)$ male. In 2001 , the average age \pm standard deviation was $73.6 \pm 11.2$, with $19.4 \%$ of residents younger than 65 . Residents of VA nursing homes were found to have high levels of functional dependence; $87 \%$ had some ADL limitation, $76 \%$ had limitations on two or more measures, and $55 \%$ had dependence in all four areas. Dependence was most commonly identified in mobility ( $79.5 \%$ of residents), followed by toileting $(74.5 \%)$, transfer $(69.2 \%)$, and eating $(64.4 \%)$. In addition, $25.2 \%$ of residents had at least a $70 \%$ service-connected disability, and on average, residents had been admitted to the nursing home $326.6 \pm 582.2$ days before their assessment.

Residents also had high levels of psychiatric morbidity. In the 6 months before their assessment, $17.9 \%$ of residents received a SMI diagnosis; $12.2 \%$ had schizophrenia as their primary SMI condition, $2.9 \%$ had bipolar disorder, and $2.7 \%$ had other psychoses. Dementia diagnoses were recorded in the records of $22.4 \%$ of residents. Of all residents, $2.8 \%$ were diagnosed with dementia and SMI diagnoses, $11.3 \%$ with substance abuse or dependence, and $3.2 \%$ with depression.

Table 1 presents resident characteristics, by SMI and dementia diagnosis status. Patients are categorized by diagnosis: SMI without comorbid dementia, dementia without comorbid SMI, dementia and SMI, or neither. Groups varied significantly on all measures. Residents with SMI alone were younger and more likely to be female, to be $70 \%$ or more service connected, to have had longer stays, and to have comorbid depression, although less likely to have comorbid substance abuse/dependence or functional dependence than residents with dementia alone.

Descriptive results regarding behaviors are presented in Table 2 by SMI and dementia category. For each of the three measures, residents with SMI or dementia were more likely to have some behavior problems than residents with neither condition. Residents with SMI without comorbid dementia had the highest prevalence of verbally disruptive behavior $(29.3 \%)$, those with dementia without comorbid SMI had the highest prevalence of physically aggressive behavior $(19.2 \%)$, and those with SMI and comorbid dementia had the highest prevalence of socially inappropriate behavior $(62.2 \%)$.

In sensitivity analyses, whether individuals diagnosed with dementia with delusional features $(290.12,290.42)$ yet who did not have an SMI diagnosis differed from other residents without SMI but with dementia was explored. It was found that only 22 patients without SMI were diagnosed with dementia with delusional features. In bivariate comparisons, these residents did not differ from the 1,861 individuals without SMI who received other dementia diagnoses, with the exception that the former were more likely to have received a diagnosis of depression.

\section{Multivariate Predictors of Resident Behaviors}

In separate multivariate ordinal logistic regression models, predictors of verbally disruptive behavior, physical aggression, and inappropriate behavior were evaluated. Estimated odds ratios (ORs), 95\% confidence intervals (CIs), and fit statistics are presented in Table 3. Although the assumption 


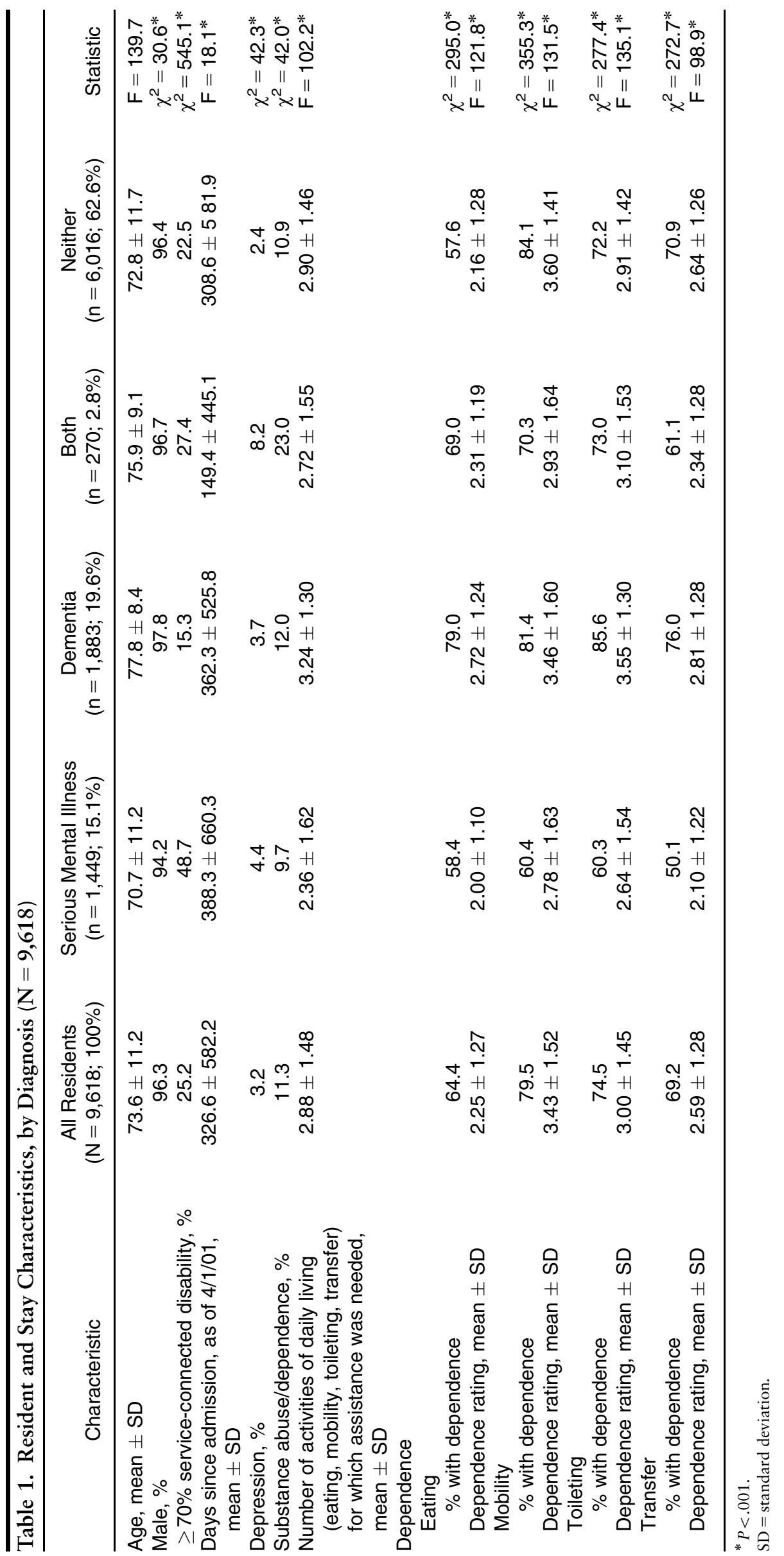


of proportional odds was not met, with large sample sizes and sets of predictors, this test will usually produce $P$-values $<.05 .^{34}$ Sensitivity analyses that dichotomized each dependent variable (some vs none) and performed multivariate logistic regressions resulted in similar patterns of association and statistical significance.

Patterns of association (Table 3 ) were generally consistent across models. In each model, residents with SMI and those with dementia were more likely to have greater behavior problems than those without such diagnoses. There were significant interaction effects between having a diagnosis of SMI and having a diagnosis of dementia. For residents with SMI, comorbid dementia was associated with a slightly lower risk of greater verbally disruptive behavior $(\mathrm{OR}=3.33 \times 2.12 \times 0.35=2.5$ vs 3.3$)$ yet increased risk of greater physical aggression $(\mathrm{OR}=3.1$ vs 2.7 ) and socially inappropriate behavior $(\mathrm{OR}=1.9$ vs 1.6$)$. Older age was associated with fewer verbally disruptive and socially inappropriate behaviors. Sex was not a significant predictor of any disruptive behaviors. Functional dependence in eating was associated with increased risk of verbal disruption and physical aggression, but it was not a significant predictor of socially inappropriate behavior. In each model, dependence in mobility was associated with less disruptive behavior. Dependence in transfer was associated with less inappropriate behavior, whereas toileting dependence was associated with increased risk in each model. Service connection of at least $70 \%$ was associated with an increased likelihood of verbal disruptions but decreased risk of inappropriate behavior. Depression was not significantly related to behavior problems, whereas substance abuse/dependence was negatively associated with physical aggression and socially inappropriate behaviors.

\section{Comparing Residents with SMI with Residents with Dementia}

Supplemental multivariate ordinal logistic regressions were performed for residents who were diagnosed with SMI (without comorbid dementia) or dementia (without comorbid SMI) $(\mathrm{n}=3,332)$. Individuals with SMI were at greater risk of verbal disruption $(\mathrm{OR}=1.49,95 \% \mathrm{CI}=1.23-1.80)$ than individuals with dementia, but risk of physical aggression and inappropriate behavior was similar for residents with SMI and residents with dementia (for SMI residents, $\mathrm{OR}=0.98,95 \% \mathrm{CI}=0.79-1.23$, for physical aggression; $\mathrm{OR}=0.96,95 \% \mathrm{CI}=0.82-1.12$, for inappropriate behavior).

\section{DISCUSSION}

This study makes contributions in three areas. First, it presented national data characterizing the population of VA nursing home residents in April 2001. Second, it evaluated the prevalence of diagnosed SMI in VA nursing home residents, including diagnoses recorded up to 6 months prior. Third, it used multivariate analyses to evaluate associations between SMI and disruptive behaviors. Supplemental analyses compared residents with SMI to those with dementia, providing a valuable reference with which to gauge these relationships.

In 2001, the VA nursing home resident population was considerably older than 15 years earlier. ${ }^{35}$ Although $31.2 \%$ 
Table 3. Ordinal Logistic Regressions of Resident Behaviors $(\mathrm{N}=9,618)$

\begin{tabular}{|c|c|c|c|}
\hline & $\begin{array}{c}\text { Model } 1 \\
\text { Verbal } \\
\text { Disruptions* }\end{array}$ & $\begin{array}{c}\text { Model } 2 \\
\text { Physical } \\
\text { Aggression }\end{array}$ & $\begin{array}{c}\text { Model } 3 \\
\text { Inappropriate }\end{array}$ \\
\hline Predictor & \multicolumn{3}{|c|}{ Odds Ratio (95\% Confidence Interval) } \\
\hline Age, 5-year units & $0.93(0.91-0.96)$ & $0.98(0.95-1.01)$ & $0.93(0.91-0.95)$ \\
\hline Male & $0.85(0.64-1.12)$ & $0.90(0.64-1.27)$ & $1.07(0.87-1.31)$ \\
\hline Day of stay, 90-day units & $1.02(1.01-1.02)$ & $1.02(1.02-1.03)$ & $1.03(1.02-1.03)$ \\
\hline \multicolumn{4}{|l|}{ Activities of daily living } \\
\hline Eating & $1.15(1.09-1.21)$ & $1.36(1.27-1.45)$ & $1.02(0.98-1.06)$ \\
\hline Mobility & $0.86(0.80-0.91)$ & $0.78(0.72-0.84)$ & $0.96(0.92-0.99)$ \\
\hline Transfer & $0.99(0.91-1.08)$ & $1.01(0.91-1.12)$ & $0.87(0.82-0.92)$ \\
\hline Toileting & $1.30(1.23-1.37)$ & $1.40(1.31-1.49)$ & $1.19(1.14-1.24)$ \\
\hline Depression & $0.77(0.54-1.08)$ & $0.89(0.59-1.34)$ & $0.86(0.68-1.07)$ \\
\hline Substance abuse/dependence & $0.91(0.75-1.11)$ & $0.73(0.56-0.95)$ & $0.75(0.66-0.86)$ \\
\hline
\end{tabular}

Model chi-square $(d f)={ }^{*} 619.6(13),{ }^{\dagger} 656.5$ (13), ${ }^{\ddagger} 392.8$ (13); $P<.001$.

Concordance score $={ }^{*} 0.69,{ }^{\dagger} 0.73,{ }^{\ddagger} 0.60$.

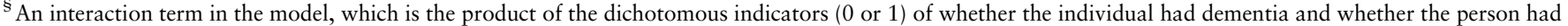
serious mental illness (SMI).

$\mathrm{SMI}=$ Serious mental illness

of VA nursing home residents were younger than 65 in 1986 , by 2001 this was true for only $19.4 \%$ of residents. Although differences have diminished, the VA nursing home population remains younger than residents of non-VA nursing homes, of which only $10 \%$ were younger than 65 in 1999. 36

Despite constituting an older resident population in 2001, the prevalence of functional dependence in VA nursing home residents was comparable with that observed 15 years earlier. ${ }^{35}$ Also, as was the case in the mid-1980s, VA nursing home residents have greater functional dependence than non-VA nursing home residents. VA nursing home residents in 2001 were more dependent in toileting $(75 \%$ vs $56 \%$ ), transfer $(69 \%$ vs $29 \%$ ), and eating $(64 \%$ vs $47 \%$ ) than participants in the 1999 National Nursing Home Survey. ${ }^{36}$

It was found that $17.9 \%$ of VA nursing home residents had a diagnosis of SMI. This was higher than the 13\% estimated prevalence among all U.S. nursing home patients in $1985 .^{26}$ It is unclear whether this difference is specific to the VA setting, reflects general population trends (as a growing number of older individuals with SMI require nursing home services), or is a function of this study's comprehensive examination of all health system diagnosis data in the prior 6 months. What is clear is that individuals with SMI represent a substantial subset of the VA nursing home resident population.

Fewer residents with dementia $(22.4 \%)$ were identified than have been found among the general nursing home resident population, in which nearly half of all residents may have dementia. ${ }^{16,20}$ Several factors may explain this difference. First, the VA nursing home resident population remains younger than the non-VA resident population. Although differences have diminished in recent years, the VA continues to care for a younger, more functionally disabled nursing home population. Methodological and definitional differences may also partially explain the lower prevalence of dementia found in this study. Diagnosis data may be a less sensitive means of identifying dementia than techniques used in other studies. Moreover, studies may vary in their definitions of dementia. Clinicians may disagree on whether certain diagnoses constitute dementia, such as other/unspecified senile psychotic conditions, other/unspecified organic brain syndromes, and amnestic syndrome. These conditions were not included in the diagnostic criteria; instead, criteria were restricted to more definite diagnostic indications. With a more diffuse set of diagnostic indicators, the assessed prevalence of dementia would be greater. In sensitivity analyses that included these conditions within the definition of dementia, the pattern of study results remained the same, but the prevalence of dementia rose to $33.3 \%$ of residents.

The goal of this study was to evaluate possible associations between diagnosed SMI and disruptive behaviors in VA nursing home residents. Evidence of significant positive associations for each category of behavior problems was found. Additionally, it was found that diagnosed dementia was positively associated with disruptive behaviors. Of residents with SMI, those with comorbid dementia had slightly lower risk of verbal disruptions than those without dementia but greater risk for physical aggression and socially inappropriate behavior.

VA nursing home residents with SMI and those with dementia were more likely than other residents to exhibit disruptive behaviors. Direct comparisons between residents with dementia (and without SMI) and those with SMI (and without comorbid dementia) indicate that SMI residents are more likely to be verbally disruptive yet are not different in their risks of physical aggression or socially inappropriate behaviors. 
Several concerns should be noted regarding this study. First, the analysis was limited to actual residents of VA nursing homes. It was not known whether nursing home residents with SMI differ substantially from individuals with SMI who require nursing homes services but have not yet received access to such services. However, prior studies indicate that seriously mentally ill nursing home residents may have worse impairment and behaviors than similarly diagnosed individuals in community settings ${ }^{28}$ and that elderly schizophrenia patients in VA nursing homes do not differ from state psychiatric hospital inpatients in the frequency and severity of verbal and physical aggression. ${ }^{29}$ Therefore, it is believed that behavioral problems in current VA nursing home residents may be representative of those in the broader population of individuals with SMI who require the level of services that nursing homes provide.

Second, it is unclear to what extent these results may be generalized to non-VA nursing home settings. Although differences have diminished, VA nursing home residents remain younger and more functionally disabled than non-VA nursing home residents. ${ }^{35}$ The vast majority of VA nursing home residents are male $(96.3 \%)$, as opposed to non-VA nursing homes, where only $38.4 \%$ of residents are male. ${ }^{36}$ In these analyses, sex was not a significant predictor of behavior problems. This may be due to having relatively few female residents in the study. Behavior dynamics may differ in less homogeneously male nursing home settings. Moreover, social and organizational dynamics in VA nursing homes settings may differ from non-VA settings, affecting behavior evaluation and management.

Finally, these analyses were limited to evaluations of associations between diagnosis variables and problem behaviors. It was not possible to distinguish whether an individual received a diagnosis as a consequence of problem behaviors or whether behavior problems were identified as a consequence of diagnostic decisions.

This study began with the question: How different are nursing home residents with SMI? It was found that VA nursing home residents with SMI were more likely than those without SMI or dementia to have behavior problems; risks for residents with SMI were comparable with those for residents with dementia. Although residents with SMI were more likely to have greater verbal disruption problems, they did not differ from residents with dementia in terms of physical aggression or inappropriate behavior.

These findings have important implications for research and policy relating to the need for, access to, and organization of nursing home care for individuals with SMI. This study demonstrates the high level of need for nursing home services among the SMI population. Nearly one in five VA nursing home residents included in the study were diagnosed with SMI. Although younger and less functionally dependent than residents without SMI, they nonetheless had substantial functional dependence and psychiatric morbidities. As the number of older individuals with SMI continues to increase, public and private health systems face important challenges in caring for this vulnerable population.

Concerns that individuals with SMI may lack appropriate access to nursing home care due to perceived greater risks of behavioral problems, stigma associated with SMI, and misgivings about facility capacities to address behavior problems motivated this study. The findings address these potential barriers by informing providers, who should reassess their concerns regarding individuals with SMI. By demonstrating their general comparability with residents with dementia in terms of disruptiveness, this study could diminish stigma associated with SMI in nursing home settings. Further research is needed to better understand concerns that these individuals may continue to present to nursing home providers and so to improve access to needed services and quality of care for all residents.

The PASARR requirements were meant to avoid inappropriate placements of individuals with SMI into nursing home settings, but it is critical to assure that nursing home policies also ensure appropriate access to nursing home services for individuals with SMI who require the level of physical health services that nursing homes provide.

Finally, by demonstrating the high prevalence of SMI in current nursing home residents, this study underscores the need to improve the organization and delivery of care in nursing homes. There are no clear answers as to how to best organize nursing home services to meet the care needs of individuals with SMI. For example, it is unclear whether individuals with SMI should be segregated from other residents, in special care units or geropsychiatry facilities, or integrated into larger nursing home resident populations. Just as there has been considerable research and clinical attention focused on behavioral problems of residents with dementia, it is also important to evaluate and inform clinical practices and staff capacities for meeting the complex needs of residents with SMI.

In particular, it is important for facilities to engage in consultation with geriatric psychiatrists, to develop staff training and expertise in mental health, and to maintain referral relationships with psychiatric care facilities. These may enhance capacities to meet the needs of individuals with SMI while reducing the stigma and anxiety associated with these psychiatric disorders.

\section{REFERENCES}

1. Everitt DE, Fields DR, Soumerai SS et al. Resident behavior and staff distress in the nursing home. J Am Geriatr Soc 1991;39:792-798.

2. Rossberg JI, Friis S. Staff members' emotional reactions to aggressive and suicidal behavior of inpatients. Psychiatr Serv 2003;54:1388-1394.

3. Beck C, Rossby L, Baldwin B. Correlates of disruptive behavior in cognitively impaired elderly nursing home residents. Arch Psychiatry Nurs 1991;5:281-291.

4. Gubrium J. Living and Dying at Murray Manor. New York: St. Martin's Press, Inc, 1975.

5. Castle NG, Mor V. Physical restraints in nursing homes: A review of the literature since the Nursing Home Reform Act of 1987. Med Care Res Rev 1998;55:139-170

6. Mosher-Ashley PM, Turner BF, O'Neill D. Attitudes of nursing and rest home administrators toward deinstitutionalized elders with psychiatric disorders. Community Ment Health J 1991;27:241-253.

7. Jervis LL. Contending with problem behaviors in the nursing home. Arch Psychiatr Nurs 2002;16:32-38.

8. McGrew KB. Residents with severe mental illnesses: How nursing homes respond. J Gerontol Soc Work 1999;31:149-168.

9. Borson S, Reichman WE, Coyne AC et al. Effectiveness of nursing home staff as managers of disruptive behavior. Am J Geriatr Psychiatry 2000;8:251-253. 10. Public Law 100-203, 1987.

11. Public Law 104-315, 104th Congress, October 1996.

12. Mechanic D, McAlpine DD. Use of nursing homes in the care of persons with severe mental illness: 1985-95. Psychiatr Serv 2000;51:354-358.

13. Younger Nursing Facility Residents with Mental Illness. Preadmission Screening and Resident Review (PASRR) Implementation and Oversight (OEI-05-9900700). Washington, DC: Office of Inspector General, U.S. Department of Health and Human Services, 2001. 
14. Borson S, Loebel JP, Kitchell M et al. Psychiatric assessments of nursing home residents under OBRA-87: Should PASARR be reformed? J Am Geriatr Soc 1997;45:1173-1181.

15. Lombardo NE. Barriers to Mental Health Services for Nursing Home Residents. Washington, DC: American Association of Retired Persons, 1994.

16. Krauss NA, Altman BM. Characteristics of nursing home residents - 1996 (MEPS Research Findings No. 5. AHCPR Publication No. 99-0006). Rockville, MD: Agency for Health Care Policy and Research, 1998.

17. Tariot PN. General Approaches to Behavioral Disturbances. In: Reichman WE, Katz PR, eds. Psychiatric Care in the Nursing Home. New York: Oxford University Press, 1996, pp 10-22.

18. Goldman HH, Feder J, Scanlon W. Chronic mental patients in nursing homes: Reexamining data from the National Nursing Home Survey. Hosp Community Psychiatry 1986;37:269-272.

19. Rovner BW, Kafonek S, Filipp L et al. Prevalence of mental illness in a community nursing home. Am J Psychiatry 1986;143:1446-1449.

20. German PS, Rovner BW, Burton LC et al. The role of mental morbidity in the nursing home experience. Gerontologist 1992;32:152-158.

21. Bartels SJ, Moak GS, Dums AR. Models of mental health services in nursing homes: A review of the literature. Psychiatr Serv 2002;53:1390-1396.

22. Allen-Burge R, Stevens AB, Burgio LD. Effective behavioral interventions for decreasing dementia-related challenging behavior in nursing homes. Int $\mathrm{J}$ Geriatr Psychiatry 1999;14:312-232.

23. Beck C, Frank L, Chumbler NR et al. Correlates of disruptive behavior in severely cognitively impaired nursing home residents. Gerontologist 1998; 38:189-198

24. Centers for Medicare and Medicaid Services. Institutions for Mental Diseases [on-line]. Available at: www.cms.hhs.gov/medicaid/services/imd.asp Accessed August 27, 2004.
25. Bartels SJ, Levine KJ, Shea D. Community-based long-term care for older persons with severe and persistent mental illness in an era of managed care. Psychiatr Serv 1999;50:1189-1197.

26. Strahan G, Burns BJ. Mental illness in nursing homes. Vital Health Stat 13 1991;Feb:1-65.

27. Lamberti JS, Tariot PN. Schizophrenia in nursing home patients. Psychiatric Ann 1995;25:441-448.

28. Bartels SJ, Mueser KT, Miles KM. A comparative study of elderly patients with schizophrenia and bipolar disorder in nursing homes and the community. Schizophr Res 1997;27:181-190.

29. Bowie CR, Moriarty PJ, Harvey PD et al. Aggression in elderly schizophrenia patients: A comparison of nursing home and state hospital residents. J Neuropsychiatry Clin Neurosci 2001;13:357-366.

30. Jackson WE, Spector WD, Rabins PV. Risk of behavior problems among nursing home residents in the United States. J Aging Health 1997;9:451472 .

31. Spector WD, Jackson ME. Correlates of disruptive behaviors in nursing homes: A reanalysis. J Aging Health 1994;6:173-184.

32. Schneider DP, Fries BE, Foley WJ et al. Case mix for nursing home payment: Resource utilization groups, version II. Health Care Financing Review Annual Supplement. Spec no. 39-52, 1988.

33. Stokes ME, Davis CS, Koch GG. Categorical Data Analysis Using the SAS System. Cary, NC: SAS Institute Inc, 1995.

34. Allison PD. Logistic Regression Using the SAS System: Theory and Applications. Cary, NC: SAS Institute, Inc, 1999.

35. Mehr DR, Fries BE, Williams BC. How different are VA nursing home residents? J Am Geriatr Soc 1993;41:1095-1101.

36. Jones A. The National Nursing Home Survey: 1999 summary. Vital Health Stat 13 2002;Jun:1-116. 